\title{
Remoção cirúrgica pós-traumática de corpo estranho intracardíaco em criança
}

\author{
Surgical removal of a posttraumatic intracardiac foreign body in a child
}

Vinicius José da Silva NINA , Rachel Vilela de Abreu Haickel NINA², Vinícius Giuliano Gonçalves MENDES³, Francival Leite de SOUZA ${ }^{4}$

\section{Resumo}

Os autores relatam a remoção cirúrgica tardia (77 dias), com sucesso, de um corpo estranho intra-atrial direito (pedaço de madeira de $7 \times 1 \mathrm{~cm}$ ) após trauma torácico transfixante em uma criança de 8 anos de idade, com história de queda sobre uma cerca. A apresentação clínica era de endocardite infecciosa confirmada pela presença de massa intracardíaca no ecocardiograma. A evolução pós-operatória tem sido satisfatória. $O$ paciente encontra-se em classe funcional I (NYHA) e em completa remissão do quadro infeccioso no seguimento de 6 meses.

Descritores: Corpos estranhos, complicações. Traumatismos cardíacos, cirurgia. Traumatismos cardíacos, etiologia.
Abstract

The authors report the successful surgical removal of a foreign body ( $7 \times 1 \mathrm{~cm}$ wooden stick) from the right atrial of a 8 year-old child 77 days after a transfixing chest trauma caused by falling over a fence. The clinical presentation was infective endocarditis confirmed by the presence of an intraatrial mass at echocardiography. The postoperative course was uneventful. At 6 months follow-up, the patient is in Function Class I (NYHA) and in complete remission of infection.

Descriptors: Foreign bodies, complications. Heart injuries, surgery. Heart injuries, etiology.

\footnotetext{
1. Doutor; Chefe do Serviço de Cirurgia Cardíaca do Hospital Universitário da Universidade Federal do Maranhão.

2. Mestre; Cardiologista Pediátrica.

3. Estudante de Medicina.

4. Especialista SBC/Ecocardiografista; Chefe da Ecocardiografia.
}

Trabalho realizado no Serviço de Cirurgia Cardíaca do Hospital Universitário da Universidade Federal do Maranhão, São Luís, MA, Brasil.

Endereço para correspondência:

Vinicius José da Silva Nina. Rua Matos Carvalho, 28 - Olho D’Água São Luís - MA - CEP: 65065-270 Tel:(5598) 3226-1099 Fax: (5598) 3232-8700.

E-mail:rvnina@terra.com.br
Artigo recebido em 19 de maio de 2007 Artigo aprovado em 5 de junho de 2007 


\section{INTRODUÇÃO}

Os corpos estranhos intracardíacos pós-traumáticos são raros, e podem causar tamponamento cardíaco, sangramento, embolia, infecção, arritmia e disfunção valvar. A sua remoção pode ser cirúrgica ou por intervenção percutânea, cuja falha pode resultar em alta morbi-mortalidade [1,2]. Neste relato, os autores descrevem a retirada cirúrgica tardia de corpo estranho intracardíaco, após trauma torácico em criança.

\section{RELATODOCASO}

Criança de 8 anos do sexo masculino, 20kg, com história de 77 dias de queda de uma árvore sobre uma cerca resultando em trauma torácico perfuro-contuso no $4^{\circ}$ espaço intercostal direito. Submetida à drenagem torácica, evoluiu com prostração e sepse, sendo necessária a antibioticoterapia e nutrição parenteral. Diante da persistência da febre diária e com a demonstração ecocardiográfica de imagem ecodensa linear atrial direita, foi aventado o diagnóstico de corpo estranho intracardíaco por provável retenção de fragmento de cateter venoso central. Deu entrada em nosso serviço em mau estado geral, hipocorado, dispnéico, emagrecido, anictérico e afebril.

Ao exame do aparelho cardiovascular, o ritmo cardíaco era regular, taquicárdico, bulhas cardíacas normofonéticas, com sopro sistólico em foco mitral de 2+/6+. A freqüência cardíaca era de 120bpm e a pressão arterial de 90x60 mmHg. A radiografia de tórax mostrou campos pulmonares limpos, com área cardíaca normal. O eletrocardiograma mostrava taquicardia sinusal, sem evidências de sobrecargas e/ou hipertrofias.

O ecocardiograma transtorácico mostrou a presença de imagem ecodensa, linear, pouco móvel, localizada transversalmente no interior do átrio direito (AD), entre o septo (próximo à desembocadura do seio venoso coronariano) e a parede lateral do $\mathrm{AD}$, sugestiva de corpo estranho. Observavamse, ainda, múltiplas imagens filamentares móveis aderidas ao corpo estranho, compatível com vegetações (Figura 1).

Após obtenção do termo de consentimento livre e esclarecido da mãe, realizou-se a toracotomia mediana, permitindo observar pericardite fibrinosa difusa, com a presença de uma formação nodular, endurecida, na parede livre do $\mathrm{AD}$, firmemente aderida ao pericárdio adjacente. Após a instalação do auxílio de circulação extracorpórea (CEC) normotérmica e proteção miocárdica com cardioplegia sanguínea fria, realizou-se a atriotomia longitudinal direita, sendo possível visualizar um pedaço de madeira de 7x1cm situado ao longo do eixo das cavas, o qual se encontrava recoberto por grande quantidade de material fibrinoso, cuja extremidade distal distava $0,5 \mathrm{~cm}$ do seio coronário e a proximal, na base do apêndice atrial, aderida à parede livre do $\mathrm{AD}$ (Figura 2).
Observou-se fibrose extensa e trombos naquele local, tornando-se necessária a excisão parcial da parede atrial. Nenhuma lesão intracardíaca foi observada. Realizou-se a atriorrafia direita em dois planos com polipropileno 5-0. O procedimento cirúrgico foi concluído de maneira habitual. O tempo de CEC foi de 30 minutos e o de isquemia miocárdica de 18 minutos.

Não houve intercorrências no pós-operatório. O exame microbiológico mostrou crescimento de hifas no material fibrinoso retirado do AD. Após 14 dias do uso de fluconazol endovenoso, o paciente recebeu alta em boas condições clínicas; afebril e com melhora do estado nutricional. No seguimento ambulatorial de 90 dias, o paciente encontravase completamente livre de sintomas, em classe funcional I (NYHA) e sem evidências ao ecodopplercardiograma de lesões intracardíacas residuais.

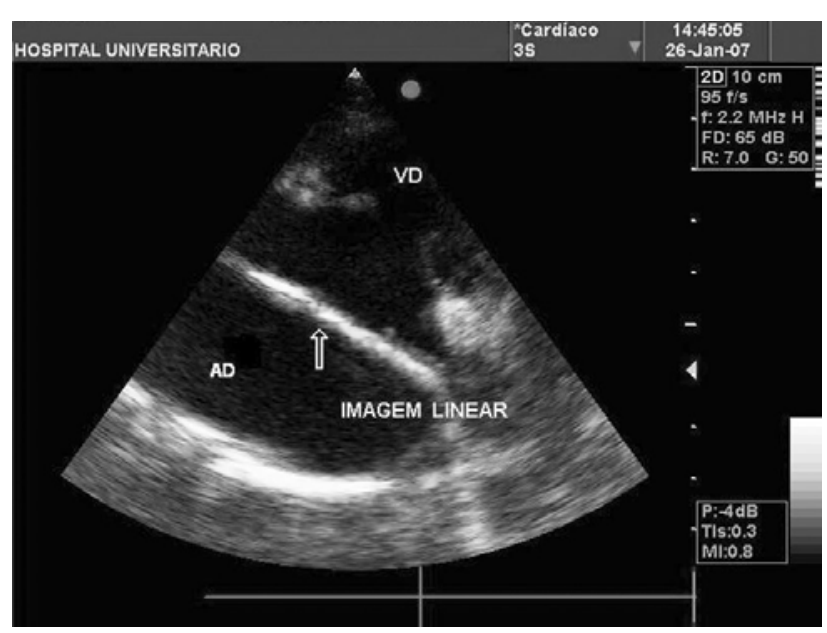

Fig. 1 - Ecocardiograma mostrando imagem linear densa intraatrial direita

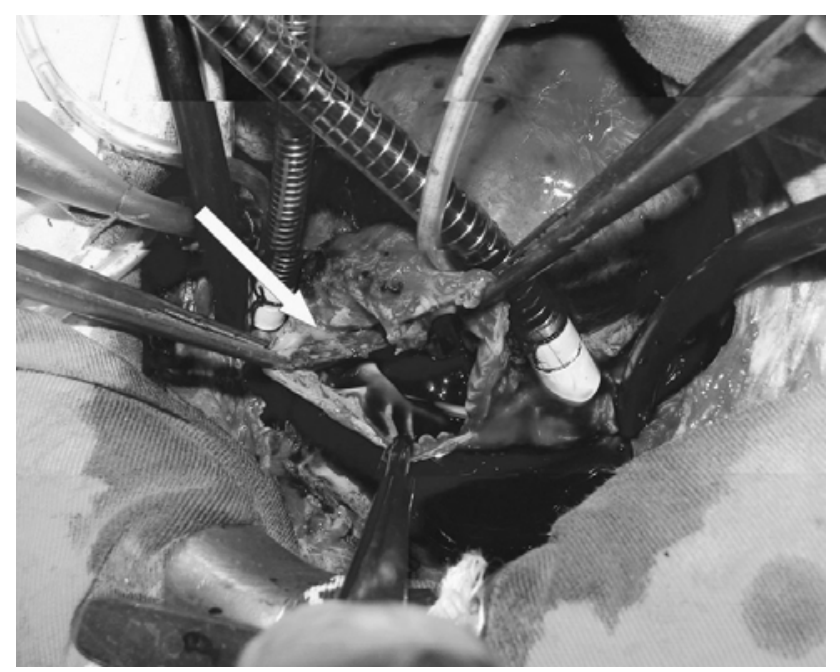

Fig. 2 - Corpo estranho intra-atrial (seta) 


\section{DISCUSSÃO}

A penetração de corpo estranho no coração pode resultar tanto da lesão direta sobre o órgão quanto de embolização secundária por penetração periférica [3]. Os corpos estranhos intracardíacos mais freqüentemente relatados na literatura são fragmentos de cateter venoso central, projéteis de arma de fogo e agulhas [1-5]. Não encontramos na literatura descrição de retenção de fragmento de madeira como corpo estranho intracardíaco.

O trauma cardíaco é um achado raro na população pediátrica. A idade média na série de Jiang et al. [1] e Actis Dato et al. [3] foi de 23,4 e 31 anos respectivamente; havendo em ambas predomínio do sexo masculino.

As manifestações clínicas da presença de um corpo estranho no coração podem ser imediatas ou tardias. A apresentação imediata é resultante de lesão cardíaca, que se acompanha de tamponamento cardíaco ou sangramento. Entretanto, se o objeto penetrante não lesar as valvas ou os septos cardíacos, o paciente pode permanecer assintomático por período variável de tempo [3,4]. Os achados do presente relato são consistentes com a literatura, no que tange à ausência de sintomas agudos quando não há comprometimento septal ou do aparelho valvar pelo corpo estranho.

Deste modo, as manifestações clínicas podem variar desde ausência de sintomas até instabilidade hemodinâmica grave. Em decorrência disto, o diagnóstico pode ser suspeitado mediante após o trauma ou, incidentalmente, durante a investigação de outra condição quando o corpo estranho permanece "silencioso". Particularmente naquela situação em que ele se aloja no miocárdio, mas não penetra na cavidade cardíaca ou permanece apenas no espaço pericárdico [1,3].

Os sintomas mais comuns são febre, pericardite, derrame pericárdico, arritmia, trombose e ansiedade. A febre pode resultar da infecção do pericárdio ou endocárdio, ou da sepse devido à presença de fragmentos contaminados como foi evidenciado neste caso, no qual o corpo estranho esteve em contato prolongado com ambos - endocárdio e o pericárdio, resultando em endocardite e pericardite [3].

Os corpos estranhos podem ser diagnosticados pela história clínica, radiologia convencional e ecocardiografia [1]. A radiografia de tórax é adequada para fazer o diagnóstico correto de corpos estranhos metálicos, entretanto, fornece apenas uma evidência qualitativa, não permite precisar a localização do objeto, não tendo sido útil na elucidação diagnóstica do presente caso $[1,4]$.

A ecocardiografia transtorácica tem sensibilidade próxima de $100 \%$ na maioria dos estudos [1,5]. Fornece informações quanto ao tamanho, localização, mobilidade e pontos de fixação dos corpos estranhos. Além disso, é um exame seguro e de execução rápida. O ecocardiograma forneceu dados suficientes para fundamentar a indicação cirúrgica do nosso caso.

O ecocardiograma transesofágico e a tomografia computadorizada devem ser reservados para os pacientes mais estáveis ou naqueles casos em que haja suspeita de corpos estranhos nos grandes vasos $[1,3]$.

A terapêutica deve ser individualizada após a avaliação do risco de se remover um corpo estranho. As opções de tratamento incluem a remoção cirúrgica, a percutânea e a terapia conservadora [1-5].

A terapêutica conservadora é reservada para os pacientes assintomáticos, sem riscos associados ou diagnosticados tardiamente após o trauma, particularmente se o corpo estranho encontra-se completamente incorporado ao miocárdio, pericárdio ou no espaço pericárdico [3,4]. O tratamento conservador requer seguimento clínico rigoroso, anticoagulação e antibioticoprofilaxia [3].

Por outro lado, pacientes assintomáticos com corpo estranho diagnosticado imediatamente após o trauma e com risco associado de infecção, embolização ou erosão das estruturas cardíacas, devem ser submetidos à remoção cirúrgica ou percutânea [2,3,5].

A remoção percutânea está indicada como tratamento inicial na presença de fragmentos de cateter, não descartando a necessidade de intervenção cirúrgica posterior [3].

A abordagem cirúrgica depende da localização do corpo estranho. Os indivíduos cujos corpos estranhos sejam parcialmente ou completamente intracavitários devem ser operados devido ao alto risco de infecção ou embolização. Entretanto, qualquer paciente sintomático, manifestando infecção, arritmia ou acidente cerebrovascular, deve ser submetido à remoção cirúrgica, independente da sua localização [1,3-5].

A presença de corpo estranho intracavitário cursando com infecção sistêmica foi o determinante da opção pelo tratamento cirúrgico no presente relato, cujo acesso foi esternotomia mediana, a qual na literatura tem-se mostrado como uma via segura dada a sua menor morbidade e permanência hospitalar quando comparada à toracotomia [3].

Os critérios para utilização de CEC na remoção cirúrgica de corpo estranho intracardíaco depende do intervalo de tempo entre o acidente e o diagnóstico, da sua localização precisa e da sua relação com a cavidade cardíaca [3].

Na série de Jiang et. al. [1], apenas dois de 11 pacientes foram submetidos à CEC, entretanto, o tempo médio entre o trauma e o diagnóstico foi de 10,1 dias. No presente relato, o tempo decorrido entre o trauma e a cirurgia foi de 77 dias, tornando qualquer tentativa de manipulação sem CEC de alto risco; visto que, o corpo estranho estava firmemente aderido ao pericárdio e ao endocárdio atrial e 
envolto por uma grande quantidade de material fibrinoso contaminado.

Em conclusão, na vigência de trauma torácico penetrante é importante investigar o comprometimento do pericárdio e do coração, para exclusão de lesão e/ou presença de corpo estranho, pois o diagnóstico precoce é fundamental para resolução cirúrgica com sucesso.

\section{REFERÊNCIAS}

1. Jiang CL, Gu TX, Wang C. Surgical treatment of posttraumatic foreign bodies in the heart or great vessels. Chin Med J. 2006;119(23):2018-20.
2. Mainar V, Picó Aracil F, Bordes P, Ruiz Ros JA, Campos Peris JV, Marín F. Percutaneous extraction of intravascular foreign bodies: a series of 38 cases. Rev Esp Cardiol. 1996;49(1):41-7.

3. Actis Dato GM, Arslanian A, Di Marzio P, Filosso PL, Ruffini E. Posttraumatic and iatrogenic foreign bodies in the heart: report of fourteen cases and review of the literature. J Thorac Cardiovasc Surg. 2003;126(2):408-14.

4. Dallan LA, Oliveira SA, Iglesias JCR, Verginelli G, Jatene AD. Remoção cirúrgica de corpo estranho transfixante cardíaco: relato de 2 casos. Rev Bras Cir Cardiovasc. 1994;9(4):220-3.

5. Yao L, Veytsman AM, Dhamee MS. Images in anesthesia: a right atrial foreign body. Can J Anaesth. 2004;51(2):173. 